\title{
Capacitação de agentes comunitários de saúde: integração entre universidade e atenção básica
}

\author{
Amanda Brait Zerbetoํ, Leonardo de Carvalho², Thaís Amanda Rossa², Daniel de Paula3
}

Resumo: O Projeto Rondon tem criado oportunidades, especialmente na saúde, para que universitários interajam com comunidades vulneráveis, socializando saberes e discutindo soluções coletivamente. O Agente Comunitário de Saúde (ACS) é um personagem fundamental na implementação do Sistema Único de Saúde (SUS), fortalecendo a integração entre os serviços de saúde e a comunidade. Mesmo com muitos avanços, a formação dos ACS permanece um desafio. Este estudo relata a experiência de um projeto de extensão na elaboração, desenvolvimento e realização de uma capacitação para ACS no município de Lindoeste, Paraná. As oficinas foram elaboradas multidisciplinarmente por acadêmicos de enfermagem, nutrição, psicologia e engenharia ambiental. Adotou-se o conceito ampliado de saúde, abordando conceitos do SUS, promoção e prevenção, educação ambiental, nutrição, humanização, além de demandas locais. A utilização de metodologias ativas e de temas relacionados à comunidade permitiram a troca de saberes, criando um espaço em que todos puderam expor suas opiniões e assim buscar soluções para os problemas locais. O desconhecimento dos ACS sobre os conceitos do SUS foi um desafio para o aprofundamento da discussão, moldando o debate muitas vezes num modelo pedagógico clássico. Um ponto positivo foi a integração e colaboração entre os ACS a partir do entendimento dos impactos que o processo de trabalho pode trazer ao município. A partir da capacitação dos ACS, ficou evidente a importância de os projetos de extensão estarem em consonância com as necessidades do território que, no presente trabalho, revelou a necessidade de elaboração e realização da educação continuada dos ACS.

Palavras-chave: Sistema Único de Saúde; Atenção Primária à Saúde; Educação Continuada; Relações Comunidade-Instituição

\section{Training community health workers: integration between university and primary} healthcare

Abstract: The Rondon Project has provided opportunities, especially in the health area, for university students to interact with vulnerable communities, share knowledge, and discuss solutions collectively. The Community Health Worker ( $\mathrm{CHW}$ ) plays a key role in implementing the Brazilian Unified Health System (Sistema Único de Saúde - SUS), strengthening the integration between health services and the community. Even with many advances, the education of $\mathrm{CHWs}$ remains a challenge. This study reports an extension project's experience in elaborating, developing, and accomplishing training for $\mathrm{CHW}$ in Lindoeste, Paraná state, Brazil. The workshops were developed in a multidisciplinary manner by nursing, nutrition, psychology, and environmental engineering students. The expanded health concept was adopted, addressing SUS concepts, health promotion, prevention, environmental education, nutrition, humanization, and local demands. The use of active learning methods and community-related themes allowed for knowledge sharing, creating an environment where everyone could express their opinions and seek solutions to local problems. CHW's lack of knowledge about SUS concepts was a challenge to deepen the discussion, often shaping the debate in a traditional way. A positive point was the integration and collaboration among $\mathrm{CHW}$, which were carried out in understanding the value of the collaborative workflow for their community. This experience highlighted the importance of the extension project being in tune with the demand of the territory, which in the present work revealed the need to elaborate and carry out continuing education of the CHW.

Keywords: : Unified Health System; Primary Health Care; Continuing Education; Community Institutional Relations
Originais recebidos em

11 de maio de 2020

Aceito para publicação em

14 de setembro de 2020

1

Departamento de Fonoaudiologia, Setor de Ciências da Saúde, Universidade Estadual do Centro-Oeste do Paraná UNICENTRO

amandabrait@gmail.com (autora para correspondência)

2

Departamento de Enfermagem, Setor de Ciências da Saúde, Universidade Estadual do Centro-Oeste do Paraná UNICENTRO

3 Departamento de Farmácia, Setor de Ciências da Saúde, Universidade Estadual do Centro-Oeste do Paraná UNICENTRO 


\section{Introdução}

O eixo fundamental da universidade é formado pelo tripé ensino, pesquisa e extensão, dimensões que devem estar presentes de forma equivalente nas Instituições de Ensino Superior (IES) no Brasil. Esse artigo foi baseado na participação de uma universidade pública estadual no projeto Rondon. Iniciado em 1968 e de iniciativa do governo federal, o projeto Rondon tem como escopo de atuação dois grandes objetivos: a formação do jovem universitário como cidadão e o desenvolvimento sustentável em comunidades vulneráveis (Decreto Presidencial n. 9.848 de 25 de junho de 2019). As IES devem propor ações com caráter de extensão que contribuam para o desenvolvimento sustentável das comunidades e para o desenvolvimento e o fortalecimento da cidadania do estudante universitário, o bem-estar social e a qualidade de vida nas comunidades carentes, usando as habilidades universitárias (Ministério da Defesa, 2019).

As ações do projeto Rondon são desenvolvidas por universitários que participam voluntariamente de capacitações de multiplicadores locais. No retorno à universidade, docentes e discentes trazem um aprendizado que, submetido à reflexão teórica, é acrescido àquele conhecimento (Domingos et al., 2016).

$\mathrm{Na}$ área da saúde, em especial, o projeto Rondon tem criado oportunidades para que os estudantes universitários interajam com as comunidades vulneráveis, socializando seus saberes e produzindo soluções inovadoras e duradouras. Em 2019, pela primeira vez, as operações se basearam em documento assinado em 2015 na Assembleia Geral da Organização das Nações Unidas (ONU), "Transformando Nosso Mundo: a Agenda 2030 para o Desenvolvimento Sustentável", que abrange as dimensões econômica, social e ambiental (United Nations, 2015; Ministério da Defesa, 2019).

As ações de saúde costumam abranger profissionais do Sistema Único de Saúde (SUS), por proporcionarem uma enriquecedora troca de experiências entre profissionais formados com os profissionais em formação. No presente trabalho é relatada uma das ações realizadas na área da Saúde na operação Yaguaru do Projeto Rondon: capacitação com Agentes Comunitários de Saúde (ACS).

O ACS é integrante da Atenção Básica (AB), que envolve um conjunto de ações de saúde individuais, familiares e coletivas, considerando a pessoa em sua singularidade e inserção sociocultural (Ministério da Saúde, Portaria de Consolidação n. 2, de 28 de setembro de 2017). O ACS é um personagem fundamental na implementação do SUS, fortalecendo a integração entre os serviços de saúde da AB e a comunidade (Oliveira et al., 2017). Nas atribuições do ACS constam a identificação de situações de risco, orientação para famílias e comunidade e encaminhamento de casos e situações de risco identificados para outros membros das equipes de saúde. A atuação do ACS possui papel complementar no planejamento e implementação de ações de saúde localmente, encaminhando informações do território para a equipe de Saúde da Família (Maciazeki-Gomes et al., 2016; Alonso et al., 2018). No Brasil há mais de 200 mil ACS e suas práticas são alvo de diversas discussões pela posição que ocupam, simultaneamente profissional de saúde e morador da comunidade (Saffer \& Barone, 2017).

No contexto das mudanças nas práticas, na mobilização social e na integração entre a população e as equipes de saúde, a importância do ACS está na sua singularidade profissional, que transita entre a assistência social, a educação, a saúde e o meio ambiente. O Ministério da Saúde reconhece que o processo de qualificação do ACS deve ser permanente (Ministério da Saúde, 2009a). A educação continuada é uma estratégia viável para manter o ACS capacitado e comprometido com sua realidade local, de forma a contribuir com a minimização dos fatores que respondem pelos indicadores sociossanitários, favorecendo o reconhecimento das condições de vida da população, de suas necessidades e prioridades (Marzari et al., 2011). 
Mesmo com muitos avanços, a formação do ACS permanece um desafio. Essa formação deve ser gradual e permanente, e realizada pelos estados e municípios em parceria com as Escolas Técnicas do SUS, considerando o contexto de trabalho do ACS e as demandas locais. A responsabilidade dessa formação é do gestor, e não deve estar vinculada especificamente a um profissional, devendo haver corresponsabilização de todos os membros da equipe de saúde da família no acompanhamento e reorientação das ações (Ministério da Saúde, 2016a). O presente estudo teve como objetivo relatar a experiência de um projeto de extensão na elaboração, desenvolvimento e realização de oficinas para ACS no município de Lindoeste, Paraná.

\section{Procedimentos Metodológicos}

As oficinas de capacitação para os ACS foram desenvolvidas como parte do Projeto Rondon do Ministério da Defesa, "Operação Yaguaru". A Operação ocorreu entre os dias 16 de janeiro e 2 de fevereiro de 2020, em doze municípios da região Oeste do estado do Paraná (Edital No 79/2019 do Ministério da Defesa), dentre esses, o município de Lindoeste, o qual foi o centro das ações relatadas no presente trabalho. A universidade participou do projeto de extensão com quinze acadêmicos de graduação, dos cursos de Agronomia, Enfermagem, Engenharia Ambiental, Engenharia Florestal, Fonoaudiologia, Jornalismo, Nutrição, Pedagogia e Psicologia, e três professores coordenadores das áreas de Farmácia, Fonoaudiologia e Geografia, compondo duas equipes correspondentes aos Conjunto "A" (áreas de Cultura, Direitos Humanos e Justiça, Educação e Saúde) e Conjunto "B" (áreas de Comunicação, Tecnologia e Produção, Meio Ambiente e Trabalho). As oficinas relatadas nesse artigo foram coordenadas pelo Conjunto "A". Entretanto, como adotou-se o conceito ampliado de saúde, acadêmicos do Conjunto " $B$ " também contribuíram para a realização das atividades.

Na primeira etapa do Projeto Rondon, as IES submeteram as propostas de trabalho de acordo com as exigências (Edital № 79/2019 do Ministério da Defesa). As IES foram informadas dos municípios em que desenvolveriam as atividades e, a partir desse momento, iniciou-se o contato com os representantes de Lindoeste. No período de 3 a 9 de novembro de 2019 foi realizada a viagem precursora com o objetivo de visitar o município, e ajustar a proposta de trabalho com as lideranças municipais e a prefeitura, de forma a atender às reais necessidades da comunidade local. Na fase seguinte foi divulgado um edital interno na universidade para a seleção dos acadêmicos. Posteriormente, as oficinas foram elaboradas com a supervisão dos professores coordenadores. As oficinas de capacitação dos ACS foram desenvolvidas por dois acadêmicos de Enfermagem dos últimos anos de graduação, com a participação de acadêmicos de Nutrição, Psicologia e Engenharia Ambiental. As temáticas das oficinas foram selecionadas conjuntamente com gestores da área da saúde do município na viagem precursora, e ajustadas por meio de contatos telefônicos e mensagens por meio digital. Durante a realização das oficinas, os ACS mostraram-se interessados em temas que não haviam sido previamente incluídos no planejamento, e por ser a proposta construída conjuntamente e com base no diálogo, esses temas foram incluídos nos próximos encontros.

Lindoeste foi fundada em 1989, com população estimada em 5.363 habitantes em 2010 (Instituto Paranaense de Desenvolvimento Social, 2020). Possui duas Unidades Básicas de Saúde (UBS) - ESF, contando com dezesseis ACS. Uma das demandas relatadas na viagem precursora por representantes da saúde foi a formação inicial e educação continuada dos ACS, pois muitos desses profissionais estavam há pouco tempo na função, e não haviam concluído o curso de formação inicial de ACS. Essa necessidade foi considerada no planejamento do projeto de extensão. Os participantes foram dispensados das visitas domiciliares e outras funções nas UBS no horário das oficinas.

As oficinas ocorreram em cinco dias consecutivos (segunda à sexta), das oito às doze horas, com intervalo de quinze minutos, totalizando vinte horas de carga horária. Foram realizadas em uma sala de grupo de uma UBS/ESF do município. As cadeiras foram posicionadas em formato de roda com a finalidade de proporcionar 
o diálogo e a troca de experiência entre todos os participantes. No planejamento, além dos temas abordados nas oficinas, foram empregadas metodologias ativas (Borges \& Alencar, 2014; Carabetta Jr, 2016; Horntvedt et al., 2018) que estimulassem a participação de todos na conversa. A Tabela 1 apresenta as oficinas realizadas.

Para a coleta de dados, as informações foram registradas em diário de campo e os depoimentos dos participantes foram gravados em áudio. Ao final de cada dia foram produzidos relatórios sobre as oficinas realizadas. Foram analisados qualitativamente os relatórios, diários de campo e depoimentos dos ACS. Os depoimentos dos ACS e registros das falas, expressões e gestuais dos profissionais nos diários de campo foram analisados com base na análise de discurso (Gill, 2002; Gerhardt \& Silveira, 2009). Para manter o sigilo, os depoimentos dos ACS foram identificados por números, por exemplo "ACS 1".

Tabela 1. Oficinas de Extensão com Agentes Comunitários de Saúde.

\begin{tabular}{|c|c|c|c|}
\hline Dia & Oficinas & $\begin{array}{l}\text { Graduandos } \\
\text { responsáveis }\end{array}$ & Metodologia(s) utilizadas \\
\hline \multirow{3}{*}{$1^{\circ}$} & $\begin{array}{l}\text { SUS: históricos, diretrizes e } \\
\text { pilares }\end{array}$ & \multirow{3}{*}{ Enfermagem } & \multirow{3}{*}{$\begin{array}{c}\text { Aula dialogada, } \\
\text { problematização, e discussão } \\
\text { em grupos focais }\end{array}$} \\
\hline & Equipe mínima da $A B$ & & \\
\hline & ACS e suas funções & & \\
\hline \multirow[b]{2}{*}{$2^{\circ}$} & $\begin{array}{l}\text { Territorialização e visita } \\
\text { domiciliar }\end{array}$ & \multirow[b]{2}{*}{ Enfermagem } & \multirow[b]{2}{*}{$\begin{array}{c}\text { Discussão baseada em textos e } \\
\text { videos disparadores }\end{array}$} \\
\hline & $\begin{array}{l}\text { Reconhecimento das } \\
\text { necessidades da população } \\
\text { geral }\end{array}$ & & \\
\hline \multirow{3}{*}{$3^{\circ}$} & $\begin{array}{l}\text { Reconhecimento das } \\
\text { necessidades da população } \\
\text { específica }\end{array}$ & \multirow{3}{*}{ Enfermagem } & \multirow{3}{*}{$\begin{array}{l}\text { Aula dialogada e aprendizado } \\
\text { em pares }\end{array}$} \\
\hline & $\begin{array}{l}\text { Promoção e prevenção de } \\
\text { saúde }\end{array}$ & & \\
\hline & Estratificação do risco familiar & & \\
\hline \multirow[b]{2}{*}{$4^{\circ}$} & Saneamento básico & \multirow[b]{2}{*}{$\begin{array}{l}\text { Engenharia } \\
\text { Ambiental }\end{array}$} & \multirow[b]{2}{*}{$\begin{array}{l}\text { Aula dialogada e } \\
\text { problematização }\end{array}$} \\
\hline & $\begin{array}{l}\text { Repensar, reduzir, reutilizar, } \\
\text { reciclar e recusar }\end{array}$ & & \\
\hline \multirow{3}{*}{$5^{\circ}$} & Alimentação saudável & Nutrição & \multirow{3}{*}{$\begin{array}{l}\text { Discussão em grupos focais, } \\
\text { reflexão guiada sobre morte e } \\
\text { luto; roda de conversa }\end{array}$} \\
\hline & Humanização & $\begin{array}{l}\text { Psicologia e } \\
\text { Enfermagem }\end{array}$ & \\
\hline & Cuidados paliativos & Psicologia & \\
\hline
\end{tabular}




\section{Relato de Experiência e Discussão}

Os resultados são apresentados e discutidos seguindo a ordem cronológica de realização das oficinas.

\section{$1^{\circ}$ dia - SUS e a equipe multidisciplinar de saúde}

No primeiro dia de oficina o tema discutido foi o SUS, pois muitos ACS não realizaram o curso básico quando assumiram a função, e considerando a necessidade de todos tomarem conhecimento sobre o sistema no qual estão inseridos. A capacitação baseou-se no curso introdutório para ACS (Ministério da Saúde, 2016b). Foram abordados os seguintes tópicos: histórico do SUS, surgimento, princípios organizativos e doutrinários, hierarquia, objetivos, eixos de ação, controle social e dimensão do sistema. Outro ponto trabalhado foi a composição mínima da equipe da $A B$ (médico, enfermeiro, ACS e técnico/auxiliar de enfermagem), debatendo as funções de cada profissão, a importância do trabalho em conjunto, e aprofundando a discussão sobre as atribuições e o papel do ACS.

Em formações com profissionais do SUS, o sistema está entre os primeiros assuntos abordados, e a abertura da oficina com este assunto teve dois objetivos: (i) situar os ACS dentro da Rede de Atenção à Saúde (RAS), em que o papel do ACS é fundamental, por ser elemento de identificação e tradução da realidade social da comunidade (Ministério da Saúde, 2016b), o que o torna um pilar para que a AB seja a porta de entrada do SUS (Oliveira et al., 2017); e (ii) verificar o conhecimento prévio sobre a dimensão da saúde pública.

Durante a realização das oficinas, optou-se pela adoção de práticas pedagógicas que aproximem a teoria da prática e problematizem situações cotidianas do trabalho. Seguindo as etapas de observação da realidade, pontos-chave, teorização, hipótese de solução e aplicação na realidade, as vivências proporcionadas, a partir da proposta pedagógica, foram significativas, e possibilitaram aos participantes repensar e reconstruir suas próprias práticas. No primeiro dia de oficina, foi perceptível o pouco conhecimento dos participantes sobre o SUS. O desconhecimento da maioria dos ACS sobre os conceitos principais do SUS foi um desafio para o aprofundamento da discussão, que assumiu em alguns momentos um caráter mais informativo por parte dos extensionistas, moldando o debate num modelo pedagógico clássico, no qual os universitários tiveram papel ativo e os ACS ficavam passivos. Esta dificuldade diminuiu o potencial da troca de saberes entre os envolvidos, troca essa que faz encontro entre os saberes populares e científicos, produzindo tecnologias sociais inovadoras e criativas para responder aos desafios sociais, econômicos e políticos apresentados pela sociedade (Lobato et al., 2013).

O uso de projeção de lâminas, juntamente com as outras dinâmicas empregadas (quiz, elaboração de cartazes, bingo, debate final) possibilitou aos participantes a troca de informações sobre os temas abordados. A utilização de diferentes recursos pedagógicos otimizou as chances de compreensão e formação de opinião sobre o assunto tratado. A escolha por diversificar o modo de apresentação corrobora com a ideia das metodologias ativas em tornar o participar mais prazeroso e facilitar a absorção das informações (Venturini, 2018).

O primeiro dia obteve avaliação positiva pelos agentes. "Estão de parabéns, muito mesmo! Até então eu não havia recebido nenhuma capacitação sobre o trabalho do ACS, eu estava trabalhando sem entender a lógica do SUS, sem entender a lógica por trás do meu trabalho e agora eu já estou tendo uma boa noção das coisas". (ACS 1).

Outro ponto positivo a ser destacado foi a integração e colaboração entre os ACS a partir do entendimento da importância dos impactos do processo de trabalho na saúde. Era de conhecimento prévio dos extensionistas a polarização política, devido ao nascimento do município ser resultado da fusão de dois distritos, Cielito Lindo e Alvorada do Oeste. Este afastamento social também se mostrou presente no comportamento e interação 
entre os ACS. A cidade conta com duas UBS/ESF, uma em cada extremidade do município, correspondendo aos antigos distritos. Com essas duas equipes de agentes trabalhando sem um engajamento em conjunto, houve espaço para a polarização política afetar a visão sobre o trabalho do outro. Foi evidente a falta de conhecimento que cada equipe tinha sobre a atuação da outra, sendo verbalizadas críticas à forma e quantidade de cadastramento de usuários que a outra UBS realizava.

Este cenário, apesar de adverso, serviu como oportunidade para reforçar a ideia do trabalho em equipe em prol da população. Com o desenvolvimento das oficinas nos cinco dias de capacitação, ouvindo os relatos de cada um e realizando dinâmicas grupais, que incentivaram a integração entre os dois lados, foram perceptíveis as mudanças na relação entre os ACS, gerando um o clima de descontração e integração.

\section{$2^{\circ}$ e $3^{\circ}$ dias - Reconhecendo as necessidades de saúde}

Nos segundo e terceiro dias de capacitação foram abordados os conceitos primordiais para a desenvolvimento do trabalho dos ACS, os quais envolvem o reconhecimento das necessidades de saúde da população em geral e das populações especificas, como hipertensos, diabéticos, idosos, mulheres e crianças.

Para conhecimento das necessidades de saúde da população, o ACS dispõe de algumas ferramentas de trabalho abordadas nesses dias: a visita domiciliar, territorialização e a estratificação do risco familiar, segundo a escala de Coelho e Savassi (2004). Esses conceitos primordiais para o trabalho de um ACS são de suma importância, pois de acordo com o Ministério da Saúde (2016), o agente é o elo entre os serviços de saúde e a comunidade (Maciazeki-Gomes et al., 2016). Portanto, é papel do ACS conhecer a sua população, o território onde ela está inserida, e como o contexto social pode interferir na vida das pessoas e, consequentemente, em seus cuidados relacionados à saúde.

O papel dos extensionistas foi discutir a função dos ACS e entender as dificuldades que tinham para exercêlas. Para melhorar o processo de trabalho dos ACS é importante o reconhecimento das necessidades de saúde da comunidade, para criação e fortalecimento de vínculos com os serviços de saúde e planejamento de ações no território.

Os ACS avaliaram as oficinas da seguinte forma:

"Eu achei bem produtivo, foi muito interessante o que a gente aprendeu, gostei bastante, muito aprendizado sobre a questão das famílias, que a gente tem que analisar o meio deles e não só ver a pessoa em si, mas pra ver como ela vive" (ACS 1).

"Eu achei bem interessante, pois muitos assuntos já aprendemos, mas acabamos esquecendo por não usar no dia a dia; e também aprendemos muitas coisas diferentes como diabetes, pé diabético. Eu, por exemplo, desconhecia. Eu acho que a interação entre vocês (extensionistas) "com nós" (ACS) está boa, muito interessante (ACS 2).

A troca de conhecimentos entre extensionistas e ACS foi muito produtiva nos segundo e terceiro dias, quando abordado o levantamento de necessidades da população e do território abrangido pelo município. Algumas das ferramentas de trabalho, tais como a territorialização e a estratificação do risco familiar, já eram adotadas no cotidiano dos agentes, contudo não eram articulados com a teoria.

A ACS 3, que recebeu a primeira capacitação para seu trabalho com o projeto de extensão, relatou "

Eu gostei bastante da capacitação, é minha primeira formação nos cinco meses que estou como ACS, está sendo muito interessante porque convivo com muito idoso, muito hipertenso, poucos diabéticos, mas temos que saber quais são os riscos, falar pra não tomar tanta medicação, fazer exercícios físicos, uma alimentação melhor. Eu adorei, bem interessante, eu aprendi muito, foi muito bom". 
Conforme os relatos dos participantes, bem como dos paradigmas observados referentes ao conhecimento técnico-científico dos ACS, o papel do projeto de extensão foi se mostrando fundamental na elucidação das funções dos ACS e para reflexão sobre como lidar com as dificuldades do trabalho. Rever a real função e o papel estratégico que os ACS desempenham dentro da ESF é decisivo para dimensionamento de ações de saúde dentro da comunidade (Alonso et al., 2018).

A formação complementar dos ACS mostra-se indispensável. Grossman-Kahn et al. (2019) evidenciaram que entre os obstáculos enfrentados pelos ACS estava "a falta de oportunidades para aprender e avançar no papel de ACS". Paralelamente, Alonso et al. (2018) ressaltaram que a percepção dos ACS sobre a formação recebida é insuficiente, o que leva a lacunas em seus conhecimentos sobre questões teóricas que poderiam auxiliar no trabalho diário. A falta de clareza de suas funções pode levar os ACS a executarem funções que não são de seu encargo.

\section{$4^{\circ}$ dia - Saúde Ambiental}

No quarto dia de oficinas foram abordadas as temáticas sobre saneamento básico, lógica dos cinco R's (Repensar, Reduzir, Reutilizar, Reciclar e Recusar), compostagem orgânica e destinação de embalagens. Apesar do tema não constar nos materiais de base na capacitação para ACS, os extensionistas optaram por desenvolver essa temática por seguirem o modelo dos determinantes sociais de saúde (Dahlgren \& Whitehead, 1991), o qual aponta que as condições ambientais interferem no processo de saúde-doença dos indivíduos. A segunda motivação para essa escolha foi a Vigilância em Saúde Ambiental, componente da Vigilância em Saúde no Brasil, regulamentada em 2001, com foco em ações que proporcionam o conhecimento e a detecção de mudanças nos fatores determinantes e condicionantes do meio ambiente que interferem na saúde humana (Ministério da Saúde, Portaria de Consolidação n. 2, de 28 de setembro de 2017).

A Saúde Ambiental é uma problemática social atual em virtude da crise mundial do meio ambiente. O debate com temas atuais aproxima os participantes de uma realidade mais palpável, na qual eles podem se situar, e perceber que fazem parte da situação. A partir deste encontro, que ofereceu ideias e informações sobre tal problema, o ACS pôde se tornar mais confiante para tomar decisões e aplicá-las em sua prática, trazendo, assim, uma autonomia para resoluções de conflitos (Barbosa \& Moura, 2014) e aumentando a potencialidade de alterar a realidade social local.

Durante as oficinas, algumas situações da cidade, tais como a falta de coleta de recicláveis, foram discutidas. A maioria dos apontamentos dos ACS foram questionamentos que necessitavam de resposta por parte da prefeitura ou até mesmo do governo estadual. Nesses momentos, os extensionistas sugeriam resoluções individuais e locais entre as comunidades do município. Também foi reforçada a importância da cobrança, denúncia e exposição da realidade aos poderes públicos. Mesmo com a dificuldade encontrada no quarto dia, o debate foi proveitoso e bem aceito pelos ACS, que participaram, debateram, questionaram e contaram experiências com muita propriedade.

"Achei o material bom, foi muito aproveitado, onde tiramos muitas dúvidas de coisas que não sabíamos. Onde achamos muito importante as informações para a prática do dia a dia." (ACS 4).

"A capacitação foi muito boa, aprendi bastante coisa, como por exemplo fazer a compostagem dos resíduos. É muito importante os ACS terem os conhecimentos sobre as questões ambientais, pois a gente tem um atendimento completo para a população."(ACS 5).

\section{$5^{\circ}$ dia - Guia alimentar, humanização e cuidados paliativos}

O último dia foi reservado para o debate sobre o guia alimentar, com ênfase na classificação dos alimentos (in natura, processados, ultraprocessados e minimamente processados), escolha e combinações de alimentos, o 
ato de comer e seus impactos diretos e indiretos na saúde. O tema não costuma estar presente na capacitação de ACS, porém, acredita-se no potencial de os "olhos da unidade de saúde" entenderem o impacto da alimentação na promoção e prevenção em saúde, especialmente em doenças de alta incidência no Brasil, incluindo hipertensão sistêmica, diabetes melitus tipo 2, gastrite e obesidade.

Esse tema foi bem avaliado pelos ACS. "Achei importante falar de temas tão facilmente esquecidos como a nutrição com o corpo [...]" (ACS 6). E participaram ativamente das atividades de dinâmicas propostas. "[...]também gosto de como tudo é bem... dinâmico, deu pra conseguir entender."(ACS 7).

A segunda temática do dia, humanização, foi um momento para relembrar a importância de enxergar o usuário como outro ser humano, repleto de necessidades sociais, assim como os próprios agentes. A proposta principal foi sensibilizar os ACS quanto a maneiras de reverter o quadro de mecanicismo, automatismo e tecnicismo, inerentes às relações de trabalho, a partir de uma nova interação entre os atores - usuários, profissionais e gestores (Ramos et al., 2018). Esta temática também serviu como momento introdutório sobre o último tema: cuidados paliativos. A escolha do tema ocorreu devido à crescente evolução no número de portadores de doenças crônicas não-transmissíveis, tais como diabetes, asma e hipertensão arterial, as quais são responsáveis por $70 \%$ das mortes mundiais e afetam com maior impacto os países em desenvolvimento (Ministério da Saúde, 2014).

Os usuários que apresentam comorbidades necessitam de maior atenção profissional, e por isso são os que mais buscam os serviços de saúde (Malta et al., 2017). Devido a características fisiopatológicas e bioquímicas, as doenças crônicas possuem um perfil degenerativo acentuado que torna seus portadores mais suscetíveis à necessidade de cuidados paliativos. Pensando na prevalência dessa população, que aumentará devido ao crescimento da população idosa no Brasil, torna-se relevante que todos os profissionais de saúde possam atuar em situações paliativas.

Mesmo sendo um tema delicado, o debate foi rico e proveitoso. "Achei importante falar de temas tão facilmente esquecidos como a nutrição com o corpo e também com a ideia de que morremos e devemos viver o dia da morte, nunca parei pra pesar nisso e muita gente não gosta, né? Então achei importante."(ACS 6).

A dificuldade do último encontro foi de caráter emocional. Com o passar dos dias, laços foram construídos entre os extensionistas e os ACS, o que contribuiu com o processo de troca de saberes e a construção de um ambiente seguro e pedagógico. Com o estabelecimento de vínculo ao longo da semana, na discussão sobre morte e cuidar surgiram relatos pessoais sobre perda de familiares e amigos próximos e luto, desviando o caráter cientifico da conversa para um clima de aconchegos e desabafos. O último momento da capacitação foi a entrega dos certificados e a avaliação. Os ACS relataram aspectos positivos do projeto, o qual proporcionou momentos de aprendizado, reflexão e troca de experiências.

Os extensionistas avaliaram o projeto como uma "oportunidade de troca de saberes, experiência e valores com a população, possibilitando um grande enriquecimento pessoal e profissional" (Extensionista 1). "A vivência me fez refletir muito sobre o ser enfermeiro dentro de uma equipe multidisciplinar e sobre o verdadeiro trabalho em equipe. Participar de um projeto de extensão, como estudante, me mostrou a importância da comunicação entre a universidade e a comunidade, e a interdependência da extensão, pesquisa e ensino" (Extensionista 2).

A Política Nacional de Educação Permanente em Saúde (PNEPS), instituída no ano de 2004, representa um marco para a formação e trabalho em saúde no País (Ministério da Saúde, 2018). As políticas públicas de saúde brasileiras, ora fundamentadas nas diretrizes do SUS, têm demonstrado importante papel para desencadear mudanças no processo de educação dos profissionais da saúde. A proposta contida na PNEPS, por exemplo, assume a regionalização da gestão do SUS, como base para o desenvolvimento de iniciativas qualificadas para o enfrentamento das carências e necessidades do sistema nacional de saúde (Ministério da Saúde, 2009b; 
2018). Neste contexto, vislumbramos o papel das atividades extensionistas realizadas com os ACS, tendo como base a regionalização das discussões em busca de soluções para problemas da comunidade, porém com iniciativas oriundas dos conhecimentos adquiridos na universidade.

No que concerne à Educação Permanente em Saúde (EPS), a definição assumida pelo Ministério da Saúde se configura como aprendizagem no trabalho, onde o aprender e o ensinar se incorporam ao cotidiano das organizações e ao trabalho. A EPS se baseia na aprendizagem significativa e na possibilidade de transformar as práticas profissionais, e acontece no cotidiano do trabalho. Nesse contexto, a EPS - como instrumento viabilizador de análise crítica e constituição de conhecimentos sobre a realidade local - precisa ser pensada e adaptada, portanto, às situações de saúde em cada nível local do sistema de saúde (Ministério da Saúde, 2018).

\section{Considerações Finais}

A capacitação realizada com os ACS por universitários voluntários demonstrou a importância de projetos de extensão estarem em consonância com as necessidades do território, que no presente trabalho revelou a necessidade de elaboração e realização da educação continuada com os ACS.

A educação continuada é prevista nos documentos do SUS, por ser fundamental na atualização e intercâmbio entre os diversos membros das RAS, e a universidade mostrou-se uma parceira para a realização dessas ações.

As oficinas foram baseadas no conceito ampliado de saúde e abordaram temas como histórico e princípios do SUS, composição e função da equipe da $A B$, promoção e prevenção de saúde, reconhecimento das necessidades da população, saúde ambiental, saúde alimentar, humanização e cuidados paliativos. A utilização de metodologias ativas e de temas relacionados ao contexto do território permitiram a troca de saberes entre os participantes, criando um espaço em que todos puderam expor suas opiniões, e assim buscar soluções para as dificuldades relatadas. Ambas as equipes de ACS puderam conhecer o trabalho uma da outra; com o desenvolvimento das oficinas, ouvindo os relatos de cada um, realizando dinâmicas grupais e incentivando a integração entre os dois lados, foi perceptível a mudança na relação entre eles e o sentimento de integração.

Por meio dos relatos dos ACS e dos extensionistas, que avaliaram essa experiência como uma oportunidade de troca entre a universidade e o serviço do SUS, ressalta-se a importância do tripé ensino, pesquisa e extensão. A universidade é uma parceira do SUS e deve realizar ações que auxiliem na construção e aprimoramento do sistema de saúde. Os serviços de saúde também têm um papel fundamental na formação universitária, para que os graduandos conheçam a realidade dos serviços do SUS e se tornem profissionais mais preparados para o trabalho em rede.

\section{Agradecimentos}

Agradecemos a todos os ACS que integraram as oficinas e aos graduandos que participaram como voluntários do Projeto Rondon, à Pró-Reitoria de Extensão e Cultura da Universidade pelo suporte administrativo na execução das atividades, e ao coordenador do Conjunto $B$, responsável pela visita precursora da operação.

\section{Contribuição de cada autor}

Os autores LC e TAR participaram da execução das atividades; os autores ABZ e DP supervisionaram as atividades de formação dos ACS; o texto final foi escrito pelos autores ABZ, LC, TAR e DP e os autores ABZ e DP realizaram a revisão crítica e aprovação final para publicação. 


\section{Referências}

Alonso, C. M. C., Béguin, P. D., \& Duarte, F. J. C. M. (2018). Work of community health agents in the Family Health Strategy: Meta-synthesis. Revista de Saúde Pública, 52(14), 1-13.

Barbosa, E. F. \& Moura, D. G. (2014). Metodologias ativas de aprendizagem no ensino de engenharia. Proceedings of International Conference on Engineering and Technology Education, Cairo, 13. [S. I.: s. n.].

Borges, T. S. \& Alencar, G. (2014). Metodologias ativas na promoção da formação crítica do estudante: O uso das metodologias ativas como recurso didático na formação crítica do estudante do ensino superior. Cairu em Revista, 3(4), 119-143.

Carabetta Jr, V. (2016). Metodologia ativa na educação médica. Revista de Medicina, 95(3), 113-121.

Coelho, F. L., \& Savassi, L. C. M. (2004). Aplicação de escala de risco familiar como instrumento de priorização das visitas domiciliares. Revista Brasileira de Medicina de Família e Comunidade, 1(2), 19-26.

Dahlgren, G., \& Whitehead, M. (1991). Policies and strategies to promote social equity in health. Stockholm: Institute for Future Studies.

Domingos, R., Silva, L. R. B., Rodrigues, L. A., Laporte, F. H., Rigoni, A., \& Beltrame, R. T. (2016). Sistema JRONDON: registro e organização de atividades extensionistas em operações do Projeto Rondon. Extensio: Revista Eletrônica de Extensão, 13(21), 105-115.

Gerhardt, T. E., \& Silveira, D. T. (2009). Métodos de pesquisa. Porto Alegre: UFRGS.

Gill, R. (2002). Análise de Discurso. In M. W. Bauer, \& G. Gaskell (Eds.), Pesquisa qualitativa com texto, imagem e som: Um manual prático. (pp. 244-270). Petrópolis: Vozes.

Grossman-Kahn, R., Schoen, J., Mallett, J.W., Brentani, A., Kaselitz, E., \& Heisler, M. (2019). Challenges facing community health workers in Brazil's family health strategy: A qualitative study. International Journal of Health Planning and Management, 33(2), 309-320.

Horntvedt, M. T., Nordsteien, A., Fermann, T., \& Severinsson, E. (2018). Strategies for teaching evidence-based practice in nursing education: A thematic literature review. BMC Medical Education, 18(1): 172.

Instituto Paranaense de Desenvolvimento Social. (2020). Caderno Estatístico Município de Lindoeste. Curitiba: IPARDES 49p. Recuperado de http://www.ipardes.pr.gov.br/Pagina/Cadernos-municipais

Lobato, P. L. M., Abranches, M., \& Rodrigues, T. V. A. (2012). Indissociabilidade ensino, pesquisa e extensão no projeto Rondon ${ }^{\circledR}$ Minas Resíduos Sólidos. Anais do Seminário de Extensão Universitária, Belo Horizonte, 7, Belo Horizonte: PUC Minas. Recuperado de: http://www1.pucminas.br/documentos/forext_02.pdf

Maciazeki-Gomes, R.C., Souza, C.D., Baggio, L., \& Wachs, F. (2016). The work of the community health worker from the perspective of popular health education: Possibilities and challenges. Ciência \& Saúde Coletiva, 21(5), 1637-1646.

Malta, D. C., Bernal, R. T. I., Lima, M. G., Araújo S. S. C., Silva, M. M. A., Freitas, M. I. F., \& Barros, M. B. A. (2017). Doenças crônicas não transmissíveis e a utilização de serviços de saúde: análise da Pesquisa Nacional de Saúde no Brasil. Revista de Saúde Pública, 51(Supl. 1): 4s.

Marzari, C. K., Junges, J. R. \& Selli, L. (2011). Agentes comunitários de saúde: Perfil e formação. Ciência \& Saúde Coletiva, $16(1), 873-880$.

Ministério da Saúde (2009a). O trabalho do agente comunitário de saúde. Brasília: Ministério da Saúde.

Ministério da Saúde (2009b). Política Nacional de Educação Permanente em Saúde. Brasília: Ministério da Saúde.

Ministério da Saúde (2016a). Diretrizes para capacitação de agentes comunitários de saúde em linhas de cuidado. Brasília: Ministério da Saúde,

Ministério da Saúde (2016b). Curso introdutório para agente comunitário de saúde (ACS). Brasília: Ministério da Saúde. 
Ministério da Saúde (2018). Política Nacional de Educação Permanente em Saúde: O que se tem produzido para o seu fortalecimento? Brasília: Ministério da Saúde.

Oliveira, A. P. C., Gabriel, M., Dal Poz, M. R., \& Dussault, G. (2017). Challenges for ensuring availability and accessibility to health care services under Brazil's Unified Health System (SUS). Ciência \& Saúde Coletiva, 22(4), 1165-1180.

Ramos, E. A., Kattah, J. A. R., Miranda, L. M., Randow, R., \& Guerra, V. A. (2018). Humanização na Atenção Primária à Saúde. Revista de Medicina de Minas Gerais, 28(Supl. 5): e-S280522.

Saffer, D. A., \& Barone, L. R. (2017). Em busca do comum: O cuidado do agente comunitário de saúde em saúde mental. Physis Revista de Saúde Coletiva, 27(3), 813-833.

Venturini, S. F. (2018). Uso e benefícios das metodologias ativas em uma disciplina de engenharia de produção. Revista de Iniciação Científica, 6(1), 59-74.

United Nations (2015). Transforming our world: The 2030 Agenda for sustainable development. [S. I.]: UN. Recuperado de https://sustainabledevelopment.un.org/post2015/transformingourworld

\footnotetext{
Como citar este artigo:

Zerbeto, A. B., de Carvalho, L., Rossa, T. A., \& de Paula, D. (2020). Capacitação de agentes comunitários de saúde: Integração entre universidade e atenção básica. Revista Brasileira de Extensão Universitária, 11(3), 349-359. https://periodicos.uffs.edu.br/index.php/ $\underline{\text { RBEU/article/view/11506/pdf }}$
} 